\title{
Milk Traces Detection in Cookies and Extruded Products Analyzed with Elisa Kits
}

\author{
Binaghi Maria Julieta ${ }^{1}$, Greco Carola Beatriz ${ }^{1}$, Martín Maria Eugenia ${ }^{1}$, Drago Silvina Rosa ${ }^{2}$, \\ Ronayne de Ferrer Patricia Ana ${ }^{1}$, López Laura Beatriz ${ }^{1}$
}

${ }^{1}$ Faculty of Pharmacy and Biochemistry, Universidad de Buenos Aires - University of Buenos Aires, Buenos Aires, Argentina

${ }^{2}$ Food Technology Institute, University Nacional of Litoral, Santa Fe, Argentina

Email address:

jbinaghi@ffyb.uba.ar (B. M. Julieta)

\section{To cite this article:}

Binaghi Maria Julieta, Greco Carola Beatriz, Martín Maria Eugenia, Drago Silvina Rosa, Ronayne de Ferrer Patricia Ana, López Laura Beatriz. Milk Traces Detection in Cookies and Extruded Products Analyzed with Elisa Kits. Journal of Food and Nutrition Sciences.

Vol. 4, No. 6, 2016, pp. 175-179. doi: 10.11648/j.jfns.20160406.16

Received: September 23, 2016; Accepted: October 7, 2016; Published: January 4, 2017

\begin{abstract}
Detection of milk traces was evaluated in cookies and extruded products model systems (MS) and in commercial products declaring milk presence through either the statement: "Contains..." or precautionary phrases. The aim of the study was to evaluate the performance of two different commercial ELISA kits for this purpose. Six cookies and extruded products model systems and two different batches of eight different types of commercial cookies and two types of extruded products were analyzed. The two different commercial ELISA kits used were R-Biopharm and Veratox-Neogen. In the cookies MS both kits had the same sensitivity while in the extruded products MS, the R- Biopharm kit had higher sensitivity than the Veratox-Neogen one. In the commercial samples only the snack declaring "Contains milk derivatives" showed detectable values of milk proteins. Even though all the remaining products included precautionary phrases on the label, none of them exceeded the quantification limit. The detection and quantification of the allergen will depend on the utilized kit and the treatment to which the ingredients have been submitted. Therefore, the performance of every kit should be studied for every allergen and every particular food matrix. Regarding commercial products, the analysis of more samples coming from different batches would be necessary in order to corroborate these results and verify the correct use of precautionary phrases.
\end{abstract}

Keywords: Allergens, Milk, Cookies, Extruded Products, ELISA

\section{Introduction}

Food allergy is a health problem which prevalence is rising rapidly. That is why, besides patients and their families, both health agents and the food industry are concerned and interest in this subject is increasing in an exponential way. It is well known that there are eight food groups that are responsible for $90 \%$ of food allergies, the "big eight": milk, egg, soy, wheat, peanuts, tree nuts, fish and shellfish. All of them constitute either part of the basic diet in most human population groups or may be ingredients used by the food industry to elaborate many different food products.

In the particular case of cow's milk protein allergy, it is the leading cause of allergies in infants and young children, and occurs in 2 to $3 \%$ of individuals in this group; however, at ages 3 to 5 about $85 \%$ of the children cease to show this pathology [1].

Allergenic milk proteins comprise the three casein fractions as well as beta-lactoglobulin [2].

Both milk and whey are common ingredients in processed foods. For this reason various regulations demand their declaration on labels, either when used as ingredients or present because of cross-contact [2].

According to the Argentine Food Code, all the ingredients used must be declared on the label. However, in some products undeclared protein ingredients have been detected [3]. In Argentina the regulations about the mandatory declaration of allergens in food labels will be published this year [4].

In our country, both government control organizations and food manufacturers require methodologies that enable the identification and quantification of allergenic proteins in a wide range of foods that should be controlled. Internationally, 
kits based on enzyme-linked immunosorbent assay (ELISA) or polymerase chain reaction (PCR) have been developed, both for raw and cooked foods, which allow the detection of some protein raw materials.

In the case of PCR, the cost of kits is very high; besides, adequate laboratories are required to carry out this type of analysis. At present, there are no PCR kits for detection of dairy allergens. ELISA techniques are simpler than PCR and do not require especial laboratories for food control. However, the cost of kits is also high and it is necessary to use diverse kits for the different raw materials that must be controlled [5].

The aim of this study was to evaluate the performance of two different commercial ELISA kits for the detection/quantification of milk proteins. For this purpose, two food matrices which are subjected to intense heat treatments during processing were chosen. Model systems for cookies and extruded products with low and known milk concentrations were analyzed. Besides, commercial cookies and extruded products declaring milk presence on the label, either by means of the statement "Contains..." or by precautionary phrases, were also analyzed in order to verify the correct declaration of milk allergens.

\section{Materials and Methods}

Model systems for gluten free cookies with rice flour, prepared at the Food Chemistry laboratory, Faculty of Pharmacy and Biochemistry, University of Buenos Aires and Model systems for extruded products with corn semolina, prepared at the Food Technology Institute, National University of the Littoral, were analysed, together with different types of commercial products. They were purchased randomly in supermarkets in Buenos Aires, Argentina. Two different batches of each product were tested.

\subsection{Model Systems}

Cookies: the cookies were made with the ingredients detailed in Table 1. Two kinds of cookies were prepared: C, without added allergens and CM5000, with addition of 5000 ppm of skim milk powder (SMP). The cookies were baked for 9 minutes 30 seconds in an oven at $250-255^{\circ} \mathrm{C}$. The temperature reached inside the cookies was $100^{\circ} \mathrm{C}$.

Six model systems were prepared mixing C and CM 5000 in different proportions. The model systems had: $0 ; 10 ; 50 ; 100$; 250 and 5000 ppm of SMP in cookies.

Extruded products: the extruded products were made with the ingredients detailed in Table 2. Two kinds of extruded products were prepared: E, without added allergens and EM 5000 , with addition of $5000 \mathrm{ppm}$ of skim milk powder (SMP). The extrusion conditions were: semolina moisturized to $14 \%$, Extrusor Brabender $10 \mathrm{DN}$, screw with compression ratio 3:1 and screw speed $150 \mathrm{rpm}$; extrusion temperature $160^{\circ} \mathrm{C}$.

Six model systems were prepared mixing E and EM 5000 in different proportions. The model systems had: $0 ; 10 ; 50 ; 100$; 250 and 5000 ppm of SMP in extruded products.

The skim milk powder was a commercial sample and contained 35\% total protein (Kjeldahl Method).

\subsection{Commercial Products}

Six different types of sweet cookies, crackers, cookies without salt added, fried potatoes and extruded product with cornmeal were analysed.

The list of ingredients of each sample is listed below:

*Sweet cookies: enriched wheat flour; sugar; refined bovine fat; starch; sorghum flour; high fructose corn syrup; salt, high oleic sunflower oil; chemical leavening: ammonium bicarbonate (INS 503ii), sodium bicarbonate (INS 500ii); emulsifier: soy lecithin (INS 322); artificial flavoring: vanilla, nature identical flavor: almond, natural flavor: lemon; food colorant: beta carotene (INS 160a(i)).

*Chocolate cookies: enriched wheat flour; sugar; refined bovine fat; cocoa powder; high fructose corn syrup; salt; chemical leavening: ammonium bicarbonate (INS 503ii), sodium bicarbonate (INS 500ii), mono calcium phosphates (INS 341i); food colorant: caramel (INS 150c); nature identical flavor: vanilla; emulsifier: soy lecithin (INS 322).

*Sweet cookies with quince jam: enriched wheat flour; quince jam; sugar; bovine fat; eggs; glucose syrup; salt; chemical leavening: ammonium bicarbonate (INS 503ii); emulsifier: soy lecithin (INS 322); food colorants: turmeric (INS100), carmine (INS120); artificial flavoring: vanilla.

*Cookies with vanilla flavour fortified with vitamins A, B1, B2, B3, B5, B6, B9, D, iron, zinc, for children from one year: enriched wheat flour; concentrated grape juice; high oleic sunflower oil; corn starch; plum pulp; ferrous sulphate; zinc sulphate; vitamins (A, D, E, B1, B2, B3, B5, B6, B9, B12); chemical leavening: ammonium bicarbonate (INS 503ii), sodium bicarbonate (INS 500ii); flavouring: natural vanilla.

*Cookies with wheat flour and banana, with banana flavour fortified with vitamins A, B1, B2, B3, B5, B6, B9, B12, D, iron, zinc, for children in early childhood: enriched wheat flour; concentrated grape juice; high oleic sunflower oil; corn starch; dehydrated banana mash; ferrous sulphate; zinc sulphate; vitamins (A, D, E, B1, B2, B3, B5, B6, B9, B12); chemical leavening: ammonium bicarbonate (INS 503ii), sodium bicarbonate (INS 500ii); flavouring: natural banana.

*Cookies with apple flavor fortified with vitamins A, B1, B2, B3, B5, B6, B9, D, iron, zinc, for children from one year: enriched wheat flour; concentrated grape juice; high oleic sunflower oil; corn starch; apple pulp; ferrous sulphate; zinc sulphate; vitamins (A, D, E, B1, B2, B3, B5, B6, B9, B12); chemical leavening: ammonium bicarbonate (INS 503ii), sodium bicarbonate (INS 500ii); flavouring: natural apple.

*Crackers: enriched wheat flour; bovine fat; salt; glucose syrup; yeast; chemical leavening: sodium bicarbonate (INS 500ii); emulsifier: soy lecithin (INS 322).

*Cookies without salt added: enriched wheat flour; bovine fat; malt extract; glucose syrup; yeast; chemical leavening: potassium bicarbonate (INS 501 ii); sodium bicarbonate (INS 500ii); emulsifier: soy lecithin (INS 322).

*Fried potatoes: potato; vegetable oil; salt.

*Cheese flavoured corn tubes: cornmeal tubes (cornmeal, modified starch, sugar, salt, acidity regulator: INS 500ii, emulsifier: INS 471); vegetable oil; cheese flavoring (salt, 
flavor enhancers: INS 621, INS 631, INS 627, food colorants: INS 110 (sunset yellow), INS 150a).

Product description, allergen declaration and precautionary labels of each commercial product are detailed in Table 3 . Two batches of each product were analysed.

Sample Processing

Stratified sampling of each food product was performed. All the samples were homogenized to a particle size of 35 mesh in a RetschR Grindomix GM200 mill.

Methods of Analysis

The detection and quantification of total milk proteins were determined with ELISA using Ridascreen ${ }^{\circledR}$ Fast Milk Protein from R-Biopharm and Veratox ${ }^{\circledR}$ Allergen Total Milk from Neogen. All samples were assayed in duplicate following the protocols of each kit $[6,7]$.

The detection (DL) and quantification (QL) limits for each kit were: Ridascreen ${ }^{\circledR}$ Fast milk Protein R-Biopharm DL:
$0.7 \mathrm{ppm}$ milk protein and QL: $2.5 \mathrm{ppm}$ milk protein with a quantification range of 2.5 - $67.5 \mathrm{ppm}$ milk protein; Veratox ${ }^{\circledR}$ Allergen Total Milk from Neogen DL: 1 ppm skim milk powder and QL: $2.5 \mathrm{ppm}$ skim milk powder with a quantification range of 2.5 - 25 ppm skim milk powder.

The final results were expressed in ppm milk protein (RBiopharm kit) or in ppm skim milk powder (Neogen kit).

\section{Results and Discussion}

\subsection{Model Systems with Skim Milk Added as an Allergen}

Table 1 shows the results for milk content using R-Biopharm and Veratox-Neogen kits in model systems for cookies with skim milk added as an allergen. Both kits allow milk quantification from $10 \mathrm{ppm}$ of skim milk added to cookies.

Table 1. Results obtained in the quantification of milk proteins using two ELISA kits in model systems of cookies with the addition of 0 - 5000 ppm of skim milk powder (SMP).

\begin{tabular}{llllll}
\hline Model systems & ppm SMP & $\begin{array}{l}\text { ppm milk } \\
\text { proteins }\end{array}$ & Ingredients & $\begin{array}{l}\text { Results R-Biopharm kit } \\
\text { (ppm milk proteins) }\end{array}$ & $\begin{array}{l}\text { Results Neogen-Veratox kit } \\
\text { (ppm SMP) }\end{array}$ \\
\hline CM 5000 & 5000 & 1750 & & $>67.5$ & $>25$ \\
CM 250 & 250 & 88 & Rice flour, manioc starch, sugar, xanthan gum, & 34.9 & $>25$ \\
CM 100 & 100 & 35 & sodium bicarbonate, margarine, lemon juice, & 21.7 & $>25$ \\
CM 50 & 50 & 18 & vanilla extract, water, skim milk powder. & 15.7 & 9.7 \\
CM 10 & 10 & 4 & Rice flour, manioc starch, sugar, xanthan gum, & 6.4 \\
C & 0 & 0 & sodium bicarbonate, margarine, lemon juice, & $<2.5$ \\
\hline
\end{tabular}

Results for milk content using R-Biopharm and Veratox-Neogen kits in model systems for extruded products with skim milk added as an allergen are shown in Table 2. R-Biopharm kit allows milk quantification from 10 ppm of skim milk added to extruded products while Veratox-Neogen kit quantifies from $50 \mathrm{ppm}$.

Table 2. Results obtained in the quantification of milk proteins using two ELISA kits in model systems of extruded products with the addition of 0 - 5000 ppm of skim milk powder (SMP).

\begin{tabular}{|c|c|c|c|c|c|}
\hline Model systems & ppm SMP & ppm milk proteins & Ingredients & $\begin{array}{l}\text { Results R-Biopharm kit } \\
\text { (ppm milk proteins) }\end{array}$ & $\begin{array}{l}\text { Results Neogen-Veratox } \\
\text { kit (ppm SMP) }\end{array}$ \\
\hline EM 5000 & 5000 & 1750 & & $>67.5$ & $>25$ \\
\hline EM 250 & 250 & 88 & & 25.1 & 8.8 \\
\hline EM 100 & 100 & 35 & Corn semolina, skim milk powder. & 15.4 & 6.2 \\
\hline EM 50 & 50 & 18 & & 8.4 & 2.8 \\
\hline EM 10 & 10 & 4 & & 3.9 & $<2.5$ \\
\hline $\mathrm{E}$ & 0 & 0 & Corn semolina & $<2.5$ & $<2.5$ \\
\hline
\end{tabular}

During the elaboration of both cookies and extruded products model systems it is very important to add the allergen under study before food processing. This is because afterwards, during processing, the allergen is exposed to physical or chemical conditions that might provoke changes on its structure, stability and solubility, as for example, in Maillard reaction [8, 9]. Food processing may cause allergens denaturation, hydrolysis, precipitation or aggregation to other food components [8]. For these reasons, allergens detection may be affected [9].

Our results showed differences in milk protein detection levels when comparing cookies and extruded products with both kits. In the cookies, both kits detected the allergen in the lowest analyzed concentration (10 ppm skim milk powder).
By contrast, in the extruded products only one of the kits allowed milk quantification in the $10 \mathrm{ppm}$ model system (RBiopharm kit), while the other kit (Veratox- Neogen) detected milk from the $50 \mathrm{ppm}$ model system. This could be explained by the different treatments suffered by milk proteins; they were less affected by baking than by the extrusion process which is much more intense. This is evidenced by a better allergen detection/quantification in the cookies.

During the extrusion process, proteins are subjected to very high temperatures and pressures which affect the possibility to detect these allergenic proteins. This could be caused not only by protein solubility changes but also by structural modifications which might negatively affect their recognition by the primary antibodies used, generating a lack in detection 
and/or quantification.

On the other hand, when comparing the quantitative results obtained with the two kits, both in cookies and extruded products model systems, they are very different from the real added amounts. In general, quantification below expected values is observed both in milk protein (R-Biopharm kit) and skim milk powder (Veratox-Neogen kit).

As already mentioned, during food processing (heat treatment) proteins might undergo denaturation, become less soluble and suffer changes altering antigen-antibody interactions. Protein immunoreactivity might be diminished or increased [9]. The main causes for the modifications in immunoreactivity are: inactivation or destruction of epitope structures, formation of new epitopes or better access of cryptic epitopes by denaturation of the native allergen [11]. All these changes could explain the differences between the added amounts and the measured values obtained in this paper.

Despite these limitations, the ELISA method is considered suitable for milk detection in food. It is important to bear in mind that the results obtained with this technique should not be considered quantitatively accurate, but semi-quantitative or qualitative when applied to processed foods [10].

Regarding the dissimilar values obtained with the two commercial kits, according to several investigators, they cannot be compared since there are many variables which could explain these discrepancies. These variables are: different products used to obtain the standard curve in each kit (skim milk powder or purified milk protein), diverse extraction solutions which cause variations in extracted proteins (phosphate buffers, addition of mercaptoethanol, etc.), informed units (ppm of skim milk powder or ppm of milk protein or ppm of casein), antibody specificity, antibody ability to bind to proteins according to the previous food processing [2]. Thus, in this paper it is not possible to compare results from both kits because they are informed in different units (ppm of skim milk powder or ppm of milk protein).

It would be very useful if commercial kit manufacturers harmonized the way of expressing results in order to make possible the comparison among them [12].

\subsection{Commercial Samples}

Table 3 shows the results for milk content using R-Biopharm kit in commercial samples. Only R-Biopharm kit was used for these samples because of its greater sensitivity for milk allergens detection in extruded products.

In the case of the samples of commercial cookies analyzed, even though all of them presented precautionary phrases about the possible presence of milk traces, none had values above the kit quantification limits ( $2.5 \mathrm{ppm}$ milk protein). The same situation was observed for one of the extruded products (potato chips). The other extruded product (Cheese flavoured corn tubes) showed values surpassing the highest concentration in the calibration curve $(>67.5 \mathrm{ppm}$ milk protein). Even though there were no dairy products declared in the ingredients list, the presence of milk informed in the allergens declaration was confirmed.

Table 3. Results obtained in the quantification of milk proteins using one ELISA kit in two batches of commercial cookies and extruded products.

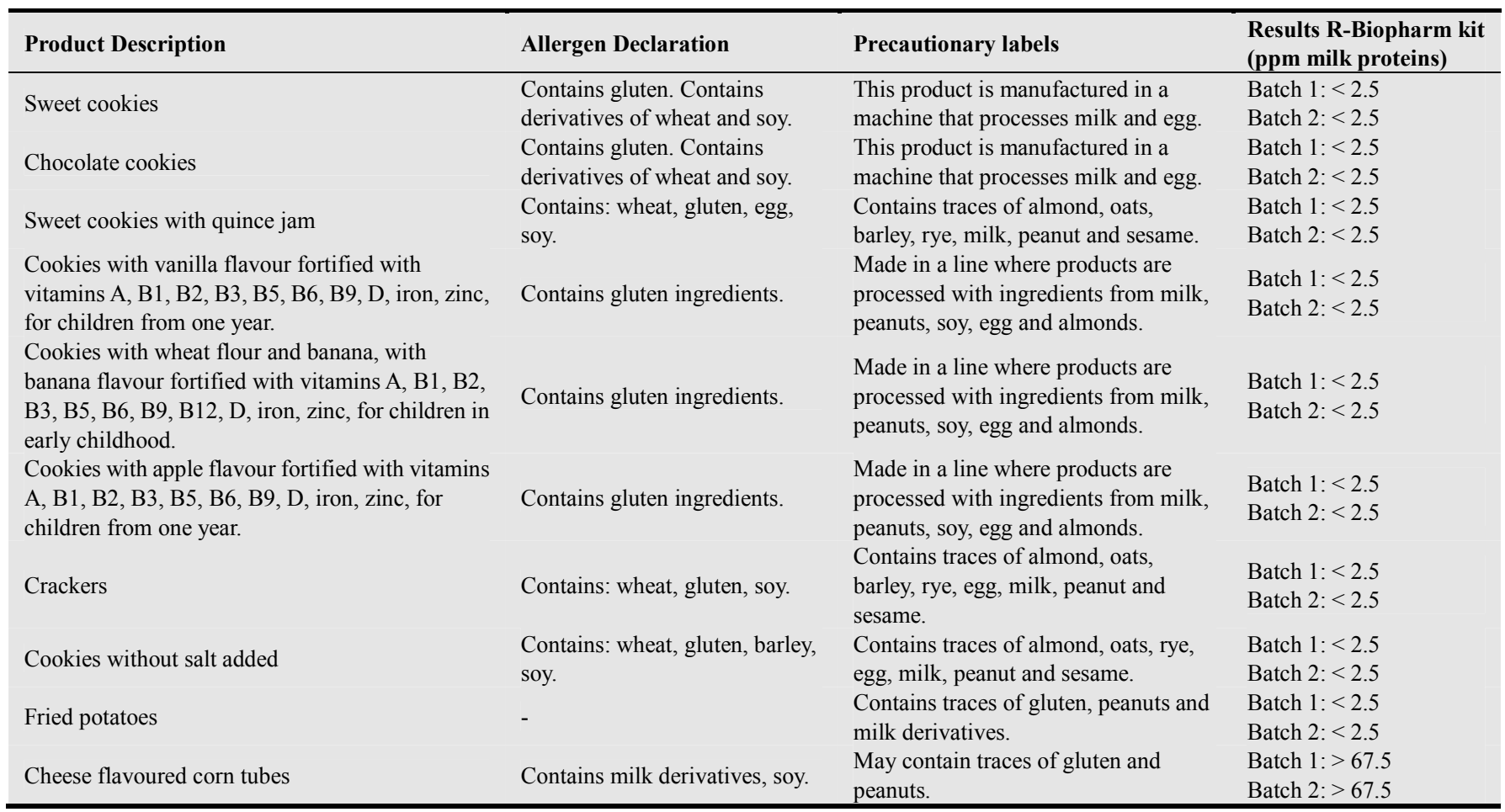

Concerning the commercial samples, the aim of the analysis in most of them (except the cheese flavoured corn tubes) was the evaluation of a possible cross-contact since none of them contained milk or dairy products. However, all of them presented precautionary phrases regarding a potential cross-contact. In the case of cheese flavoured corn tubes, it is 
presumed that "cheese flavor" would be the ingredient containing dairy products.

It is important to consider that most of these foods are usually consumed by children and, as already mentioned, in this population group cow's milk protein allergy has a high prevalence.

In order to carry out a correct allergen declaration in labels, food manufacturers should implement an effective allergen control program in their plants, taking into account concepts related to food allergens management and food safety.

Good Manufacturing Practices (GMP) aimed at assuring segregation of allergenic ingredients have improved in recent years and this is reflected in the systematic label declaration of allergens. However, much more work is needed to minimize the risk of cross-contact and to provide more safety to allergic consumers by means of veritable communication though labeling.

Faced with the lack of knowledge about the allergen levels required to provoke adverse reactions, many manufacturers have adopted a presumed "foolproof" scheme by including precautionary phrases in the labels. Even though this was initially embraced by allergic consumers as a useful practice, the increasing and inconsistent use of this type of warning has considerably diminished its impact [13]. This problem has led consumers to disregard the precautionary statements and thus has forced them to either make personal consults to food manufacturers or thoroughly reduce variety in their diets.

The presentation of precautionary phrases should be clear, unequivocal and consistent for consumers. If precautionary statements were used correctly, they could become great communication tools and decrease risk significantly. However, experience shows that a long list of allergens as potential contaminants is usually included, even though many of them never enter the food manufacturing plants. If a responsible declaration was done, the number of available foods for the affected population could increase [14].

\section{Conclusions}

The detection and quantification of the allergen will depend on the utilized kit and the treatment to which the ingredients have been submitted. Therefore, the performance of every kit should be studied for every allergen and every particular food matrix.

Regarding commercial products, all of those presenting precautionary statements about the potential presence of milk traces showed values below the kit quantification limit. The analysis of more samples coming from different batches would be necessary in order to corroborate these results and verify the correct use of precautionary phrase

\section{Acknowledgements}

This work was partially financed by Projects UBACyT 20020120100175BA and UBACyT 20020130100520BA.

\section{References}

[1] Chapman J, Bernstein L, Lee R, Oppenheimer J. 2006. Food allergy: a practice parameter. Annals of Allergy, Asthma \& Immunology. 96 (3 Suppl 2): S1-S68.

[2] Díaz Amigo C. 2010. Towards a Comprehensive Validation of ELISA Kits or Food Allergens. Case2-Milk. Food Analytical Methods. 3: 351-356.

[3] Cellerino K, Binaghi MJ, Cagnasso CE, Docena G, López LB. 2014. Milk protein detection in raw and cooked meat products using immunochemical methods. Journal of Food and Nutrition Sciences. 2(5): 236-242.

[4] Act $\mathrm{N}^{\circ} 111$ CONAL. 2016.

http://www.conal.gob.ar/actas/Acta_111.pdf Access: 06/ 09/2016.

[5] López L, Binaghi M, Greco C, Mambrín M, Valencia M. 2011. Meat species identification in cooked meat products: using SDS-PAGE as screening method. Revista Chilena de Nutrición. 38 (2): 187-196.

[6] RIDASCREEN ${ }^{\circledR}$ Fast Milk protein (Art. No.: R4652). R-Biopharm. $2016 . \quad$ Available: http://www.R-Biopharm.com/products/food-feed-analysis/alle rgens/milk/item/ridascreenfast-milk. Access: September 2016.

[7] Veratox ${ }^{\circledR}$ Total Milk (Code: 8470). Neogen, 2016. Available: http://foodsafety.neogen.com/en/veratox-total-milk Access: September 2016.

[8] Díaz Amigo C, Popping B. 2010. Analytical Testing as a Tool for the Enforcement of Future Regulatory Thresholds for Food Allergens. Journal of AOAC International. 93 (2): 434-441.

[9] Parker C, Khuda S, Pereira M, Ross M, Fu T, Fan X, Wu Y, Williams K, DeVries J, Pulvermacher B, Bedford B, Zhang X and Jackson L. 2015. Multi-allergen Quantitation and the Impact of Thermal Treatment in Industry-Processed Baked Goods by ELISA and Liquid Chromatography-Tandem Mass Spectrometry. Journal of Agricultural and Food Chemistry. 63: 10669-10680.

[10] Popping B, Diaz-Amigo C, Hoenicke K. 2010. Chapter 17 in Molecular Biological and immunological techniques and applications for food chemists. John Wiley \& Sons, Inc. Canada.

[11] Besler M., Steinhart H., Paschke A. 2001. Stability of food allergens and allergenicity of processed food. Journal of chromatography B: Biomedical Sciences and Applications. 756 (1-2): 207-228.

[12] Díaz Amigo C. 2010. Towards a Comprehensive Validation of ELISA Kits for Food Allergens. Case 1- Egg. Food Analytical Methods. 3: 344-350.

[13] Sampson MA, Munoz-Furlong A, \& Sicherer SH. 2006. Risktaking and coping strategies of adolescents and young adults with food allergy. Journal of Allergy and Clinical Immunology. 117: 1440-1445.

[14] Ward R, Crevel R, Bell I, Khandke N, Ramsay C and Paine S. 2010. A vision for allergen management best practice in the food industry. Trends in Food Science \& Technology 21: 619-625. 